\title{
PCSK9 rs7552841 is associated with plasma lipids profiles in female Chinese adolescents without posttraumatic stress disorder
}

\author{
Qiwei Guo, Yanjun Si, Mi Su, Mei Fan, Jia Lin, Nazakat H Memon, Dingzhi Fang* \\ Department of Biochemistry and Molecular Biology, West China School of Basic Medical Sciences \& Forensic Medicine, Sichuan \\ University, Chengdu, China.
}

\begin{abstract}
Summary To explain the inconsistent relationship between proprotein convertase subtilisin/kexin type 9 (PCSK9) rs7552841 and plasma lipids profiles, we hypothesized that interplays might occur among gender, PCSK9 rs7552841 and posttraumatic stress disorder (PTSD) on plasma lipids levels. To test this hypothesis, a population of 704 Chinese Han high school students was used, which had been recruited after the 2008 Wenchuan Earthquake. In this population, the plasma levels of glucose, triglyceride (TG), total cholesterol (TC), high-density lipoprotein cholesterol (HDL-C) and low-density lipoprotein cholesterol (LDL-C) had been measured by routine methods. PTSD had been assessed by the PTSD Checklist Civilian Version (PCL-C). PCSK9 rs7552841 was analyzed by polymerase chain reaction-restriction fragment length polymorphism analyses and verified by DNA sequencing. The T allele carriers had significantly higher levels of TG, TC, LDL-C, and glucose than the CC homozygotes of PCSK9 rs7552841 after the adjustment for age and BMI in the female students, but not in the male students. When PTSD was taken into consideration, the female $T$ allele carriers had significantly higher TG, TC, LDL-C and glucose than the female CC homozygotes after the adjustment for age and BMI only in the subjects without PTSD, but not in the PTSD patients. No significant differences were observed in the male students regardless of PTSD and the adjustment for age and BMI. These results suggest that PCSK9 rs7552841 is associated with plasma lipids profiles only in female adolescents, but not in male students. This association can be modified and negated by PTSD.
\end{abstract}

Keywords: PCSK9 rs7552841, genetic variation, gender, PTSD, blood lipids

\section{Introduction}

Posttraumatic stress disorder (PTSD) is a serious chronic anxiety problem (1) that develops in some people after experiencing extremely traumatic events such as serious injury, actual or threatened death, childhood abuse and natural disasters (2-4). Numerous studies indicated that individuals with PTSD exhibited higher levels of triglyceride (TG), total cholesterol (TC) and low-density lipoprotein cholesterol (LDL-C) but lower levels of high

Released online in J-STAGE as advance publication October 29, 2017.

*Address correspondence to:

Dr. Dingzhi Fang, Department of Biochemistry and Molecular Biology, West China School of Basic Medical Sciences \& Forensic Medicine, Sichuan University, Chengdu 610041, China.

E-mail: dzfang@scu.edu.cn density lipoprotein cholesterol (HDL-C) than healthy control subjects (5-7). However, Jendricko et al. (8) reported that there were no differences of plasma $\mathrm{TG}$, TC, HDL-C and LDL-C between the male war veterans with PTSD and the control subjects. Jergovic et al. (9) also reported that plasma lipids did not differ between PTSD patients and controls in individual time points. The mechanism of the discrepancy has not been elucidated yet.

In recent years, proprotein convertase subtilisin/ kexin type 9 (PCSK9) has emerged as a new, promising key therapeutic target to reduce the plasma levels of TC, especially LDL-C $(10,11)$. PCSK9, also known as neural apoptosis-regulated convertase 1 , is a serine protease mostly secreted by liver that plays a critical role in cholesterol metabolism (12). In particular, PCSK9 promotes the degradation of cell-surface LDL receptor (LDL-R), resulting in reduced clearance of 
LDL-C from circulation through a post-transcriptional mechanism (12-14). Human PCSK9 gene (PCSK9) is located on chromosome $1 \mathrm{p} 32$, expanding promoter region and 12 exons (15). Gain-of-function mutations of PCSK9 were found to increase the degradation of LDL-R, resulting in autosomal dominant hypercholesterolemia. These findings were confirmed by mice experiments using the wild-type and mutant genes such as S127R and F216L (15-17). Conversely, heterozygous patients with loss-of-function mutations had lower plasma LDL-C levels and decreases of $80-90 \%$ risk of coronary heart disease $(16,18)$. Furthermore, previous studies have demonstrated that PCSK9 is not only take part in the regulation of lipids profiles, but also associated with mental disorders. Kang et al. (19) reported that plasma levels of PCSK9 were increased in both Alzheimer's disease (AD) and mild cognitive impairment patients. It was also shown recently that $\mathrm{AD}$ patients had significantly higher levels of PCSK9 in cerebrospinal fluid than non-AD subjects (20). However, there are no existing reports about the relationship between PCSK9 and PTSD.

In addition, over 40 single nucleotide polymorphisms have been found at PCSK9. Much more recently, $P C S K 9$ rs7552841 in the intron region with a thymine $(\mathrm{T})$ to cytosine $(\mathrm{C})$ transition has been reported to be associated with statin response in European individuals (21). Significant differences of plasma levels of TG, TC and LDL-C were also observed between the $\mathrm{T}$ allele carriers and the $\mathrm{CC}$ homozygotes of PCSK9 rs7552841 in a Chinese Jing population (22). Forevermore, the distribution of the $\mathrm{T}$ allele carriers and the CC homozygotes were significantly different between hypercholesterolaemic and control subjects or between hypertriglyceridaemic and control subjects. After adjusting age, gender, body mass index (BMI), smoking and alcohol consumption, PCSK9 rs7552841 was found to be associated with not only hypercholesterolaemia but also hypertriglyceridaemia (23). On the other hand, no significant differences of plasma lipid levels were observed between the $\mathrm{T}$ allele carriers and the CC homozygotes in a Chinese Han population (22), and in 101 Chilean hypercholesterolaemic individuals before or after 10 $\mathrm{mg}$ /day of atorvastatin therapy although the T allele carrier showed a trend towards less increases of HDL-C after the treatment (24). Obviously, more studies are needed to clarify the above discrepancies of the associations reported before between PCSK9 rs7552841 and plasma lipids profiles.

To explain the discrepancies of the associations between PTSD and plasma lipids profiles, and between PCSK9 rs7552841 and plasma lipids profiles reported previously by others, we hypothesized that interplays might occur between PCSK9 rs7552841 and PTSD to influence plasma lipids profiles. The present study was to test the hypothesis. In fact, we had a population of high school students experiencing the 2008 Wenchuan earthquake, which we had explored the PTSD characteristics in 2008 and 2009, and reported them (25). We also had measured the plasma lipids profiles after sampling in 2008 (26). In the present study, we genotyped PCSK9 rs7552841 and analyzed the plasma lipids profiles in the students with different genotypes of PCSK9 rs7552841 and with or without PTSD. To our knowledge, this is the first study exploring the interaction between PTSD and PCSK9 rs7552841 on plasma lipids profiles.

\section{Materials and Methods}

\subsection{Study population}

As reported before (25), the population was from a boarding high school after the 2008 Wenchuan earthquake. The devastating earthquake occurred in the afternoon of 12 May 2008, measuring 8.0 on the Richter scale and led to 69,227 deaths, 17,923 missing and 374,643 injured. The earthquake extended about 10 thousand $\mathrm{km}^{2}$ and covered 254 towns in 21 counties, which destroyed 6.5 million houses. About 5 million people were evacuated and lived in temporary shelters after the earthquake. The school is located only 10 $\mathrm{km}$ away from the epicenter of the earthquake and was severely damaged during the earthquake. All the teaching halls and students dormitories were destroyed by the earthquake. The students lived and studied in temporary houses.

Although PTSD characteristics had been measured at 6,12 and 18 months after the earthquake (25), only the data at 6 months were used to test the hypothesis in the present study, which included 746 students from grade 11 . Among them, $737(98.8 \%)$ had completed the questionnaires evaluating their demographic characteristics and PTSD status. Recruitment criteria were understanding of the procedures involved, no history of metabolic disease and providing written consents and blood samples. The students with cardiovascular, renal, or endocrinological diseases, or diabetes, and the students who took lipid-lowering drugs or hormones, consumed alcohol and smoked were excluded. In the end, 704 students were included in the present study. They were all Chinese Han adolescents at the age of 15 to 18 years with an average of $16.86 \pm$ 0.59 years. There were 310 male students $(44.03 \%)$ and 394 female students (55.97\%). Written consents had been provided by all the participants of the study and their guardians. This study was approved by the Human Research Ethics Committee of Sichuan University, ratifying our lab to analyze not only the psychological characteristic, genetic variations and plasma lipids profiles, but also the interplays psychological characteristic, genetic variations on plasma lipids profiles (26). 


\subsection{Measurements}

The measurement instrument was composed of two parts, which was reported previously (25). The first part had been used to assess measures including demographic characteristics such as gender, age, body weight and height. Body mass index (BMI) had been calculated. The PTSD Checklist-Civilian Version (PCL-C) (27) had been used to assess PTSD in the second part, which corresponds to Diagnostic and Statistical Manual of Mental Disorders, 4th edition (DSM-IV) criteria (2). The PCL-C includes 17 selfreport items. The total score ranges from 17 to 85 . A total score of 38 was defined as the cutoff point of PTSD in this study (28). The assessments had been finished in 2008 and the existing data were used for the present study.

\subsection{Blood collection and laboratory analyses}

Twelve-hour fasting venous bloods had been sampled. The levels of plasma glucose, TG and TC had been measured using enzymatic methods. The levels of HDL-C had been determined after separated by phosphotungstate-magnesium chloride precipitation method. The levels of LDL-C had been examined using the polyvinyl sulfate precipitation method. All the samples had been measured 3 times and the average values were used for statistical analyses. All the measurements had been finished after sampling in 2008 and the existing data were used for the present study.

\subsection{DNA extraction and genotyping}

Genetic DNA had been isolated after sampling by others in the lab from peripheral blood leucocytes using a DNAout kit (Tiandz, China) and stored at $-80^{\circ} \mathrm{C}$. In the present study, the stored genetic DNA was used for the genotyping of PCSK9 rs7552841. The genotypes of PCSK9 rs7552841 were determined by polymerase chain reactionrestriction fragment length polymorphism method and verified by DNA sequencing. The following PCR primers were used for the amplifications of DNA containing PCSK9 rs7552841 locus: forward primer, 5'-AGGGAAGGGCACGGTTAG-3', reverse primer, 5'-TGCCAGTTCCTCCACCAC-3'. The PCR cycling parameters were denaturation at $95^{\circ} \mathrm{C}$ for $2 \mathrm{~min}, 30$ subsequent cycles of denaturation at $95^{\circ} \mathrm{C}$ for $30 \mathrm{sec}$, annealing at $60^{\circ} \mathrm{C}$ for $30 \mathrm{sec}$ and extension at $72^{\circ} \mathrm{C}$ for $30 \mathrm{sec}$, and a final extension at $72^{\circ} \mathrm{C}$ for $2 \mathrm{~min}$. The resulting PCR products were DNA fragments of 496 bp. The identification of the genotypes was performed by the restriction digestion with $M s p$ I. The digested products were separated by electrophoresis on $1.5 \%$ agarose gel. As a result, the digested PCR products with the CC genotype migrated as one band of $496 \mathrm{bp}$, the
TT genotype as two bands of 320 and $176 \mathrm{bp}$ and the CT genotype as three bands of 496, 320 and $176 \mathrm{bp}$.

\subsection{Statistical analyses}

The results were expressed as mean \pm standard deviation (SD) unless otherwise stated. Log power transformations were applied to the levels of TG because they were in positively skewed distribution. The genotype and allele frequencies were determined by counting. The agreement of the genotype distribution of PCSK9 rs7552841 with Hardy-Weinberg equilibrium was assessed by chi-squared goodness-of-fit tests. Differences of genotype frequencies as well as allele distribution between the male and female students were estimated using Chi-Square $\left(\chi^{2}\right)$ tests. Independentsamples $t$ tests were used to compare all the other quantitative variables between the male and female students and between the individuals with different genotypes of PCSK9 rs7552841. Covariance analyses were used to eliminate the potentially confounding bias of age and BMI on biochemical characteristics. The level of statistical significance was set at 0.05 .

\section{Results}

\subsection{Characteristics of the study population}

The levels of plasma lipids and the related metabolic variables in the study population are shown in Table 1. The male subjects were significantly older than the female subjects in the present study. The levels of BMI, TG, TC, HDL-C and LDL-C were significantly higher, but glucose was significantly lower in the female students when compared with those of the male students.

\subsection{Genotype and allele frequencies of PCSK9 rs 7552841}

The genotype and allele frequencies of PCSK 9 rs7552841 in the adolescents are presented in Table 2. No deviation was found from Hardy-Weinberg equilibrium of the distribution of the genotypes of PCSK9 rs7552841 in the study population $\left(\chi^{2}=0.17\right)$. There were no statistically significant differences in the genotype and allele frequencies between the male and female subjects. As there were limited subjects with the TT genotype, they were combined with the subjects with the CT genotype and defined as the $\mathrm{T}$ allele carriers for further analyses and presented as CT/TT in the tables.

\subsection{Prevalence of PTSD in the subjects with different genotypes of PCSK9 rs 7552841}

According to the assessment of PCL-C, the prevalence of PTSD in the students with different genotypes of 
Table 1. Characteristics of the study population

\begin{tabular}{lcccc}
\hline Variables & All & Males & Females & $p$-Value \\
\hline$n$ & 704 & 310 & 394 & -- \\
age & $16.86 \pm 0.59$ & $16.95 \pm 0.61$ & $16.78 \pm 0.56$ & $<0.001$ \\
BMI, $\mathrm{kg} / \mathrm{m}^{2}$ & $20.28 \pm 2.31$ & $19.81 \pm 2.29$ & $20.66 \pm 2.27$ & $<0.001$ \\
TG, mmol/L & $1.12 \pm 0.44$ & $0.96 \pm 0.35$ & $1.24 \pm 0.47$ & $<0.001$ \\
TC, $\mathrm{mmol} / \mathrm{L}$ & $3.58 \pm 0.57$ & $3.41 \pm 0.51$ & $3.72 \pm 0.58$ & $<0.001$ \\
HDL-C, mmol/L & $1.41 \pm 0.28$ & $1.36 \pm 0.26$ & $1.45 \pm 0.29$ & $<0.001$ \\
LDL-C, $\mathrm{mmol} / \mathrm{L}$ & $1.67 \pm 0.49$ & $1.61 \pm 0.46$ & $1.71 \pm 0.50$ & 0.011 \\
Glucose, $\mathrm{mmol} / \mathrm{L}$ & $5.07 \pm 0.44$ & $5.15 \pm 0.45$ & $5.01 \pm 0.42$ & $<0.001$ \\
\hline
\end{tabular}

a comparisons between those of the male subjects and those of the female subjects (independent-samples $t$ test).

Table 2. Genotype and allele frequencies of PCSK9 $\operatorname{rs} 7552841$ in the study population

\begin{tabular}{|c|c|c|c|c|c|}
\hline Genotype & $\begin{array}{c}\text { Total }(n=704) \\
n(\%)\end{array}$ & $\begin{array}{c}\text { Hardy-Weinberg } \\
p\end{array}$ & $\begin{array}{c}\text { Males }(n=310) \\
n(\%)\end{array}$ & $\begin{array}{c}\text { Females }(n=394) \\
n(\%)\end{array}$ & $p^{\mathrm{a}}$ \\
\hline \multicolumn{6}{|c|}{ Genotype Frequencies } \\
\hline $\mathrm{CC}$ & $510(72.4)$ & & $221(71.3)$ & $289(73.4)$ & \\
\hline $\mathrm{CT}$ & $180(25.6)$ & & $84(27.1)$ & $96(24.4)$ & 0.606 \\
\hline $\mathrm{TT}$ & $14(1.99)$ & 0.683 & $5(1.60)$ & $9(2.30)$ & \\
\hline \multicolumn{6}{|c|}{ Allele Frequencies } \\
\hline $\mathrm{C}$ & $1,200(85.2)$ & & $526(84.8)$ & $674(85.5)$ & 0.715 \\
\hline $\mathrm{T}$ & $208(14.8)$ & & $94(15.2)$ & $114(14.5)$ & \\
\hline
\end{tabular}

Data are presented as $n(\%)$. ${ }^{a}$ Male students $v s$. female students by Chi-Square tests.

Table 3. Prevalence of PTSD in the subjects with different genotypes of PCSK9 rs7552841

\begin{tabular}{|c|c|c|c|c|c|c|}
\hline \multirow{2}{*}{ Group } & \multicolumn{2}{|c|}{ All } & \multicolumn{2}{|c|}{ Males } & \multicolumn{2}{|c|}{ Females } \\
\hline & $\mathrm{CC}$ & $\mathrm{CT} / \mathrm{TT}$ & $\mathrm{CC}$ & $\mathrm{CT} / \mathrm{TT}$ & $\mathrm{CC}$ & $\mathrm{CT} / \mathrm{TT}$ \\
\hline Control & $346(67.8)$ & $136(70.1)$ & $166(75.1)$ & $71(79.8)$ & $180(62.3)^{\mathrm{a}}$ & $65(61.9)^{\mathrm{a}}$ \\
\hline PTSD & $164(32.2)$ & $58(29.9)$ & $55(24.9)$ & $18(20.2)$ & $109(37.7)$ & $40(38.1)$ \\
\hline
\end{tabular}

Data are expressed as $n(\%) .{ }^{a} p<0.05$ when compared with that of the male students (Chi-Square test).

PCSK9 rs7552841 is shown in Table 3. No significant difference of prevalence was found between the CC homozygotes and the T allele carriers in all the students, the male or the female subjects. The PTSD prevalence of female subjects was significantly higher than the male subjects in both the CC homozygotes $(p=0.002)$ and the $\mathrm{T}$ allele carriers $(p=0.008)$.

3.4. Plasma lipids profiles and the related metabolic variables of the subjects with different genotypes of PCSK9 rs 7552841

Table 4 shows age, BMI, plasma lipids and plasma glucose of the subjects with different genotypes of PCSK9 rs7552841. The female T allele carriers had higher levels of TC and LDL-C than the female CC homozygotes. After the adjustment for age and BMI, the female $\mathrm{T}$ allele carriers had significantly higher levels of TG, TC, LDL-C and glucose than the female $\mathrm{CC}$ homozygotes. However, there were no significant differences between the $\mathrm{T}$ allele carriers and the $\mathrm{CC}$ homozygotes in the male subjects regardless of the adjustment for age and BMI.

3.5. Effects of PTSD on the association of PCSK9 rs7552841 polymorphism with plasma lipids profiles and the related metabolic variables

As shown in Table 5, no significant differences were found between the $\mathrm{CC}$ homozygotes and the $\mathrm{T}$ allele carriers in the male students regardless of PTSD and the adjustment for age and BMI. However, the T allele carriers had higher levels of BMI, TG, TC, and LDL-C than the CC homozygotes in the female subjects without PTSD, but not in the female students with PTSD. After the adjustment for age and BMI, the female $\mathrm{T}$ allele carriers had significantly higher levels of TG, TC, LDL-C, and glucose than the female CC homozygotes in the students without PTSD. Still, no significant differences were observed after the adjustment for 
Table 4. Serum Lipids Profiles and the Related Metabolic Variables of the subjects with different genotypes of PCSK9 rs7552841

\begin{tabular}{|c|c|c|c|c|c|c|c|c|}
\hline \multirow{2}{*}{ Variables } & \multicolumn{4}{|c|}{ Males } & \multicolumn{4}{|c|}{ Females } \\
\hline & $\mathrm{CC}$ & $\mathrm{CT} / \mathrm{TT}$ & $p$-Value ${ }^{\mathrm{a}}$ & $\begin{array}{l}\text { ANCOVA } \\
p \text {-Value }^{\mathrm{b}}\end{array}$ & $\mathrm{CC}$ & $\mathrm{CT} / \mathrm{TT}$ & $p$-Value ${ }^{\mathrm{a}}$ & $\begin{array}{l}\text { ANCOVA } \\
p \text {-Value }^{\mathrm{b}}\end{array}$ \\
\hline$n$ & 221 & 89 & -- & -- & 289 & 105 & -- & -- \\
\hline Age, year & $16.95 \pm 0.61$ & $16.96 \pm 0.62$ & 0.950 & -- & $16.79 \pm 0.59$ & $16.76 \pm 0.49$ & 0.635 & -- \\
\hline BMI, $\mathrm{kg} / \mathrm{m}^{2}$ & $19.83 \pm 2.36$ & $19.76 \pm 2.11$ & 0.810 & -- & $20.74 \pm 2.22$ & $20.44 \pm 2.38$ & 0.254 & -- \\
\hline $\mathrm{TG}, \mathrm{mmol} / \mathrm{L}$ & $0.95 \pm 0.34$ & $0.98 \pm 0.37$ & 0.545 & 0.486 & $1.21 \pm 0.46$ & $1.31 \pm 0.47$ & 0.055 & 0.025 \\
\hline $\mathrm{TC}, \mathrm{mmol} / \mathrm{L}$ & $3.41 \pm 0.52$ & $3.41 \pm 0.48$ & 0.950 & 0.975 & $3.68 \pm 0.56$ & $3.85 \pm 0.64$ & 0.008 & 0.004 \\
\hline HDL-C, $\mathrm{mmol} / \mathrm{L}$ & $1.35 \pm 0.25$ & $1.36 \pm 0.28$ & 0.819 & 0.867 & $1.46 \pm 0.29$ & $1.43 \pm 0.28$ & 0.316 & 0.212 \\
\hline LDL-C, mmol/L & $1.62 \pm 0.48$ & $1.60 \pm 0.43$ & 0.686 & 0.717 & $1.66 \pm 0.48$ & $1.82 \pm 0.54$ & 0.004 & 0.002 \\
\hline Glucose, $\mathrm{mmol} / \mathrm{L}$ & $5.15 \pm 0.41$ & $5.14 \pm 0.52$ & 0.862 & 0.873 & $4.98 \pm 0.41$ & $5.08 \pm 0.43$ & 0.051 & 0.047 \\
\hline
\end{tabular}

${ }^{\mathrm{a}}$ Comparisons between those of the CC homozygotes and those of the T allele carriers (independent-samples $t$ test). ${ }^{\mathrm{b}}$ Analyses of covariance with the adjustment for age and BMI.

Table 5. Effects of PTSD on the association of PCSK9 rs7552841 with anthropometric and biochemical characteristics

\begin{tabular}{|c|c|c|c|c|c|c|c|c|c|}
\hline \multirow{2}{*}{ Variables } & \multirow{2}{*}{ Group } & \multicolumn{4}{|c|}{ Males } & \multicolumn{4}{|c|}{ Females } \\
\hline & & $\mathrm{CC}$ & $\mathrm{CT} / \mathrm{TT}$ & $p$-Value ${ }^{a}$ & $\begin{array}{l}\text { ANCOVA } \\
p \text {-Value }{ }^{b}\end{array}$ & $\mathrm{CC}$ & $\mathrm{CT} / \mathrm{TT}$ & $p$-Value ${ }^{\mathrm{a}}$ & $\begin{array}{c}\text { ANCOVA } \\
p \text {-Value }\end{array}$ \\
\hline \multirow[t]{2}{*}{$n$} & Control & 166 & 71 & -- & -- & 180 & 65 & -- & -- \\
\hline & PTSD & 55 & 18 & -- & -- & 109 & 40 & -- & -- \\
\hline \multirow[t]{2}{*}{ Age, year } & Control & $16.96 \pm 0.62$ & $16.93 \pm 0.66$ & 0.752 & -- & $16.78 \pm 0.58$ & $16.75 \pm 0.50$ & 0.717 & -- \\
\hline & PTSD & $16.93 \pm 0.57$ & $17.06 \pm 0.42$ & 0.384 & -- & $16.81 \pm 0.60$ & $16.78 \pm 0.48$ & 0.760 & -- \\
\hline \multirow[t]{2}{*}{ BMI, kg/m² } & Control & $19.68 \pm 2.06$ & $19.58 \pm 1.99$ & 0.727 & -- & $20.69 \pm 2.08$ & $19.97 \pm 2.31$ & 0.021 & -- \\
\hline & PTSD & $20.25 \pm 3.09$ & $20.44 \pm 2.46$ & 0.816 & -- & $20.81 \pm 2.44$ & $21.21 \pm 2.31$ & 0.371 & -- \\
\hline \multirow[t]{2}{*}{$\mathrm{TG}, \mathrm{mmol} / \mathrm{L}$} & Control & $0.94 \pm 0.28$ & $0.98 \pm 0.35$ & 0.312 & 0.265 & $1.20 \pm 0.42$ & $1.33 \pm 0.46$ & 0.036 & 0.014 \\
\hline & PTSD & $1.00 \pm 0.49$ & $0.97 \pm 0.42$ & 0.836 & 0.792 & $1.23 \pm 0.52$ & $1.28 \pm 0.49$ & 0.579 & 0.799 \\
\hline \multirow[t]{2}{*}{$\mathrm{TC}, \mathrm{mmol} / \mathrm{L}$} & Control & $3.37 \pm 0.52$ & $3.42 \pm 0.49$ & 0.506 & 0.464 & $3.67 \pm 0.64$ & $3.84 \pm 0.62$ & 0.042 & 0.031 \\
\hline & PTSD & $3.53 \pm 0.52$ & $3.36 \pm 0.41$ & 0.208 & 0.183 & $3.68 \pm 0.59$ & $3.87 \pm 0.67$ & 0.090 & 0.135 \\
\hline \multirow[t]{2}{*}{ HDL-C, mmol/L } & Control & $1.35 \pm 0.25$ & $1.35 \pm 0.28$ & 0.992 & 0.929 & $1.47 \pm 0.28$ & $1.42 \pm 0.28$ & 0.234 & 0.095 \\
\hline & PTSD & $1.37 \pm 0.26$ & $1.41 \pm 0.29$ & 0.562 & 0.411 & $1.44 \pm 0.30$ & $1.44 \pm 0.28$ & 0.907 & 0.960 \\
\hline \multirow[t]{2}{*}{ LDL-C, $\mathrm{mmol} / \mathrm{L}$} & Control & $1.59 \pm 0.47$ & $1.62 \pm 0.43$ & 0.659 & 0.588 & $1.66 \pm 0.45$ & $1.81 \pm 0.54$ & 0.041 & 0.012 \\
\hline & PTSD & $1.71 \pm 0.49$ & $1.51 \pm 0.46$ & 0.127 & 0.087 & $1.67 \pm 0.52$ & $1.85 \pm 0.57$ & 0.079 & 0.117 \\
\hline \multirow[t]{2}{*}{ Glucose, $\mathrm{mmol} / \mathrm{L}$} & Control & $5.12 \pm 0.41$ & $5.14 \pm 0.52$ & 0.804 & 0.833 & $4.98 \pm 0.37$ & $5.09 \pm 0.46$ & 0.057 & 0.041 \\
\hline & PTSD & $5.25 \pm 0.40$ & $5.16 \pm 0.56$ & 0.479 & 0.463 & $5.00 \pm 0.47$ & $5.06 \pm 0.40$ & 0.431 & 0.484 \\
\hline
\end{tabular}

${ }^{\mathrm{a}}$ Comparisons between those of the $\mathrm{CC}$ homozygotes and those of the $\mathrm{T}$ allele carriers (independent-samples $t$ test). ${ }^{\mathrm{b}}$ Analyses of covariance with the adjustment for age and BMI.

age and BMI between the T allele carriers and the $\mathrm{CC}$ homozygotes in the female students with PTSD.

\section{Discussion}

In the present study, we used the population that we had reported the PTSD characteristics (25) and had measured the lipids profiles (26). We genotyped PCSK9 rs7552841 and analyzed the plasma lipids profiles in the students with different genotypes of PCSK9 rs7552841 and with or without PTSD. To our best knowledge, these analyses have not been reported before. In fact, PTSD characteristics had been measured in the population at 6,12 and 18 months after the earthquake (25). However, only the data at 6 months were used in the present study because the purpose was to test our hypothesis that interplays might occur between PCSK9 rs7552841 and PTSD to influence plasma lipids profiles. Although the follow-up might provide more information about the changes of PTSD and lipids profiles, analyzing the interplays of course, PTSD and PCSK9 rs7552841 was not the purpose of the present study.

It has been reported that $\mathrm{PCSK} 9$ can regulate plasma levels of LDL-C, while some of the single nucleotide polymorphisms at PCSK9 are associated with plasma lipids profiles $(12-14,16,29)$. Recently, the association of PCSK9 rs7552841 polymorphism with plasma lipids has been explored in two laboratories in China and Chile (22-24). Significant differences were found by the Chinese laboratory of the plasma levels of TG, TC and LDL-C between the T allele carriers and the CC homozygotes of PCSK9 rs7552841 in a Chinese Jing population, but not in Chinese Han population (22). The results reported by the Chilean laboratory showed that there were no significant differences 
of plasma lipids between the $\mathrm{T}$ allele carriers and the CC homozygotes of PCSK9 rs7552841 in 101 Chilean hypercholesterolaemic individuals before and after $10 \mathrm{mg} /$ day of atorvastatin therapy (24). These discrepancies may be explained by ethnicities, healthy status, medication status and even the sample size because the Chilean investigation was carried out in a smaller population. However, other confounding factors such as age, BMI, gender or psychological factors were not included in all the above analyses, which have been generally accepted to be important factors associated with plasma lipids profiles (30-34). In the present study, the female students had significantly higher levels of TG, TC, HDL-C and LDL-C but lower levels of glucose than the male students in the whole study population (Table 1). These results confirm again that gender serves as an important confounding factor and influences the levels of plasma lipids. Therefore, we examined the differences of plasma lipids between the $\mathrm{T}$ allele carriers and the CC homozygotes of PCSK9 rs7552841 in the male and female students separately. To eliminate the influence of confounders such as age and BMI on plasma lipids, the adjustment was made of age and BMI. The results show that the female $\mathrm{T}$ allele carriers had significantly higher levels of TG, TC, LDL-C and glucose than the female CC homozygotes after the adjustment for age and BMI. Nevertheless, no significant differences were observed between the $\mathrm{T}$ allele carriers and the $\mathrm{CC}$ homozygotes in the male subjects regardless of the adjustment for age and BMI (Table 4). These results suggest that PCSK9 rs7552841 may interplay with gender to influence plasma lipids profiles. And therefore, PCSK9 rs 7552841 is associated with plasma lipids in a gender-dependent manner. This finding may be one of the explanations of the discrepancies reported before of the associations between PCSK9 rs7552841 and the levels of plasma lipids.

Although a biopsychosocial medical model was proposed by Engel in 1977 (35), much less efforts have been made to explore the interplays between biomedical factors and psychological factors on plasma lipids profiles. Therefore, in the present study, PTSD was selected as a psychological factor to study its interplays with gender and PCSK9 rs7552841 on the levels of plasma lipids in a biological-psychological approach. The results indicate that the female $\mathrm{T}$ allele carriers had significantly higher levels of TG, TC, LDL-C and glucose than the female $\mathrm{CC}$ homozygotes after the adjustment for age and BMI only in the subjects without PTSD, but not in the PTSD patients (Table 5). No significant differences were observed between the male $\mathrm{T}$ allele carriers and the male $\mathrm{CC}$ homozygotes regardless of PTSD and the adjustment for age and BMI. These results suggest that PCSK9 rs7552841 may interplay with not only gender but also PTSD to affect plasma levels of TG, TC, LDL-C and glucose. More specifically, the association between PCSK9 rs7552841 and plasma lipids profiles in female subjects may be modified or eliminated by PTSD. These findings may be one of the explanations that no associations were found between PCSK9 rs7552841 and the levels of plasma lipids in the same ethnicity reported before (22). Therefore, in the future study, gender and psychological factors should be taken into account when the relationship is tested between PCSK9 rs7552841 and plasma lipids profiles.

Cunningham et al. (36) reported that the mutant with the gain-of-function mutation D374Y could bind LDL receptor more tightly than the wild-type PCSK9, resulting in the increment of plasma LDL-C. On the other hand, Suzanne et al. (37) found that PCSK9 with the natural mutation F216L could increase the levels of circulating LDL-C through resisting to furin digestion and increasing plasma PCSK9 levels. In addition, some other gain-of-function mutations were also reported to increase the levels of plasma LDL-C (16,38-40). As the variation of PCSK9 rs7552841 is located at the intron region, the molecular mechanism of its effects on plasma lipids profiles cannot be the gain-offunction mutation of PCSK9. The possible mechanism may be that its mRNAs are more stable since higher stabilities of mutant intron-containing RNAs have been found to promote translation, resulting in more protein production $(41,42)$. Other mechanisms such as linkage disequilibrium should also be taken into consideration.

There were some limitations in the present study. Firstly, only adolescents were included in the present study. The metabolic characteristics of this population may be different from adults. Secondly, the plasma levels of PCSK9 and the levels of PCSK9 mRNA in liver tissues were not measured. Thirdly, other psychological factors such as depression were not included, which had been reported to be related to dyslipidemia $(43,44)$. These measurements are recommended for future studies in this field.

In conclusion, there may be interactions among gender, PTSD and PCSK9 rs7552841 on plasma lipids profiles. PCSK9 rs7552841 is associated with plasma lipids profiles in a gender-dependent manner in Chinese Han adolescents without PTSD, but not in Chinese Han adolescents with PTSD. The T allele of PCSK9 rs7552841 may be a risk factor to increase the levels of plasma TG, TC, LDL-C and glucose in healthy female subjects. This finding may provide novel insights into the regulation of lipids metabolisms by PCSK9 in younger populations, and pave the way for the precision prevention to reduce risks of CVD in adolescents, especially in a country with a quarter of the world's population.

\section{Acknowledgements}

This study was supported by the Program of Sichuan 
Province for International Cooperation and Exchanges of Sciences and Technologies (Grant no. 2017HH0074). Professor Dingzhi Fang is the recipient of the grant.

\section{References}

1. Davis LL, Suris A, Lambert MT, Heimberg C, Petty F. Post-traumatic stress disorder and serotonin: New directions for research and treatment. J Psychiatry Neurosci. 1997; 22:318-326.

2. APA. Diagnostic and Statistical Manual of Mental Disorders, DSM-IV-TR Fourth Edition. American Psychiatric Association, Washington, DC, 2000, USA.

3. Lancaster CL, Teeters JB, Gros DF, Back SE. Posttraumatic Stress Disorder: Overview of EvidenceBased Assessment and Treatment. J Clin Med. 2016; 5. pii: E105.

4. Neumeister A, Seidel J, Ragen BJ, Pietrzak RH. Translational evidence for a role of endocannabinoids in the etiology and treatment of posttraumatic stress disorder. Psychoneuroendocrinology. 2015; 51:577-584.

5. Levine AB, Levine LM, Levine TB. Posttraumatic stress disorder and cardiometabolic disease. Cardiology. 2014; 127:1-19.

6. Dennis PA, Ulmer CS, Calhoun PS, Sherwood A, Watkins LL, Dennis MF, Beckham JC. Behavioral health mediators of the link between posttraumatic stress disorder and dyslipidemia. J Psychosom Res. 2014; 77:45-50.

7. Maia DB, Marmar CR, Mendlowicz MV, Metzler T, Nobrega A, Peres MC, Coutinho ES, Volchan E, Figueira I. Abnormal serum lipid profile in Brazilian police officers with post-traumatic stress disorder. J Affect Disord. 2008; 107:259-263.

8. Jendricko T, Vidovic A, Grubisic-Ilic M, Romic Z, Kovacic Z, Kozaric-Kovacic D. Homocysteine and serum lipids concentration in male war veterans with posttraumatic stress disorder. Prog Neuropsychopharmacol Biol Psychiatry. 2009; 33:134140.

9. Jergovic M, Bendelja K, Savic Mlakar A, Vojvoda V, Aberle N, Jovanovic T, Rabatic S, Sabioncello A, Vidovic A. Circulating levels of hormones, lipids, and immune mediators in post-traumatic stress disorder - A 3-month follow-up study. Front Psychiatry. 2015; 6:49.

10. Nissen SE, Dent-Acosta RE, Rosenson RS, et al. Comparison of PCSK9 Inhibitor Evolocumab vs Ezetimibe in Statin-Intolerant Patients: Design of the Goal Achievement After Utilizing an Anti-PCSK9 Antibody in Statin-Intolerant Subjects 3 (GAUSS-3) Trial. Clin Cardiol. 2016; 39:137-144.

11. Norata GD, Ballantyne CM, Catapano AL. New therapeutic principles in dyslipidaemia: Focus on LDL and Lp(a) lowering drugs. Eur Heart J. 2013; 34:17831789.

12. Peterson AS, Fong LG, Young SG. PCSK9 function and physiology. J Lipid Res. 2008; 49:1152-1156.

13. Park SW, Moon YA, Horton JD. Post-transcriptional regulation of low density lipoprotein receptor protein by proprotein convertase subtilisin/kexin type 9a in mouse liver. J Biol Chem. 2004; 279:50630-50638.

14. Lagace TA, Curtis DE, Garuti R, McNutt MC, Park SW, Prather HB, Anderson NN, Ho YK, Hammer RE, Horton JD. Secreted PCSK9 decreases the number of LDL receptors in hepatocytes and in livers of parabiotic mice. J Clin Invest. 2006; 116:2995-3005.

15. Abifadel M, Varret M, Rabes JP, et al. Mutations in PCSK9 cause autosomal dominant hypercholesterolemia. Nat Genet. 2003; 34:154-156.

16. Benjannet S, Rhainds D, Essalmani R, et al. NARC-1/ PCSK9 and its natural mutants: Zymogen cleavage and effects on the low density lipoprotein (LDL) receptor and LDL cholesterol. J Biol Chem. 2004; 279:48865-48875.

17. Maxwell KN, Breslow JL. Adenoviral-mediated expression of Pcsk9 in mice results in a low-density lipoprotein receptor knockout phenotype. Proc Natl Acad Sci U S A. 2004; 101:7100-7105.

18. Zhao Z, Tuakli-Wosornu Y, Lagace TA, Kinch L, Grishin NV, Horton JD, Cohen JC, Hobbs HH. Molecular characterization of loss-of-function mutations in PCSK9 and identification of a compound heterozygote. Am J Hum Genet. 2006; 79:514-523.

19. Kang S, Jeong H, Baek JH, Lee SJ, Han SH, Cho HJ, Kim H, Hong HS, Kim YH, Yi EC, Seo SW, Na DL, Hwang D, Mook-Jung I. PiB-PET Imaging-Based Serum Proteome Profiles Predict Mild Cognitive Impairment and Alzheimer's Disease. J Alzheimers Dis. 2016; 53:1563-1576.

20. Zimetti F, Caffarra P, Ronda N, Favari E, Adorni MP, Zanotti I, Bernini F, Barocco F, Spallazzi M, Galimberti D, Ricci C, Ruscica M, Corsini A, Ferri N. Increased PCSK9 Cerebrospinal Fluid Concentrations in Alzheimer's Disease. J Alzheimers Dis. 2017; 55:315320.

21. Thompson JF, Hyde CL, Wood LS, Paciga SA, Hinds DA, Cox DR, Hovingh GK, Kastelein JJ. Comprehensive whole-genome and candidate gene analysis for response to statin therapy in the Treating to New Targets (TNT) cohort. Circ Cardiovasc Genet. 2009; 2:173-181.

22. Guo T, Yin RX, Huang F, Yao LM, Lin WX, Pan SL. Association between the DOCK7, PCSK9 and GALNT2 Gene Polymorphisms and Serum Lipid levels. Sci Rep. 2016; 6:19079.

23. Guo T, Yin R-X, Lin W-X, Wang W, Huang F, Pan S-L. Association of the variants and haplotypes in the DOCK 7, PCSK9 and GALNT2 genes and the risk of hyperlipidaemia. J Cell Mol Med. 2016; 20:243-265.

24. Cuevas A, Fernández C, Ferrada L, Zambrano T, Rosales A, Saavedra N, Salazar LA. HMGCR rs17671591 SNP Determines Lower Plasma LDL-C after Atorvastatin Therapy in Chilean Individuals. Basic Clin Pharmacol Toxicol. 2016; 118:292-297.

25. Zhang Z, Ran MS, Li YH, Ou GJ, Gong RR, Li RH, Fan M, Jiang Z, Fang DZ. Prevalence of post-traumatic stress disorder among adolescents after the Wenchuan earthquake in China. Psychol Med. 2012; 42:1687-1693.

26. Fan M, LiRH, Hu MS, Fang DZ. Effects of interactions between post-traumatic stress disorder with brain-derived neurotrophic factor gene Val66Met polymorphism on serum lipid profiles in adolescents. China Sciencepaper. 2015; 10:2876-2879. (in Chinese)

27. Blanchard EB, Jones-Alexander J, Buckley TC, Forneris CA. Psychometric properties of the PTSD Checklist (PCL). Behav Res Ther. 1996; 34:669-673.

28. Tian Y, Wong TK, Li J, Jiang X. Posttraumatic stress disorder and its risk factors among adolescent survivors three years after an 8.0 magnitude earthquake in China. BMC public health. 2014; 14:1073.

29. Scartezini M, Hubbart C, Whittall RA, Cooper JA, 
Neil AH, Humphries SE. The PCSK9 gene R46L variant is associated with lower plasma lipid levels and cardiovascular risk in healthy U.K. men. Clin Sci (Lond). 2007; 113:435-441

30. Wenger NK. Gender disparity in cardiovascular disease: Bias or biology? Expert Rev Cardiovasc Ther. 2012; 10:1401-1411.

31. Zheng J, Gao Y, Jing Y, Zhou X, Shi Y, Li Y, Wang L, Wang R, Li M, Xiao C, Li Y, Li R. Gender differences in the relationship between plasma lipids and fasting plasma glucose in non-diabetic urban Chinese population: A cross-section study. Front Med. 2014; 8:477-483.

32. Fall T, Hagg S, Ploner A, et al. Age- and sex-specific causal effects of adiposity on cardiovascular risk factors. Diabetes. 2015; 64:1841-1852.

33. Engebretson TO, Stoney CM. Anger expression and lipid concentrations. Int J Behav Med. 1995; 2:281-298.

34. Chikani V, Reding D, Gunderson P, McCarty CA. Wisconsin rural women's health study psychological factors and blood cholesterol level: Difference between normal and overweight rural women. Clin Med Res. 2004; 2:47-53.

35. Engel GL. The need for a new medical model: A challenge for biomedicine. Science. 1977; 196:129-136.

36. Cunningham D, Danley DE, Geoghegan KF, et al. Structural and biophysical studies of PCSK9 and its mutants linked to familial hypercholesterolemia. Nat Struct Mol Biol. 2007; 14:413-419.

37. Benjannet S, Rhainds D, Hamelin J, Nassoury N, Seidah NG. The proprotein convertase (PC) PCSK9 is inactivated by furin and/or PC5/6A: Functional consequences of natural mutations and post-translational modifications. $\mathrm{J}$
Biol Chem. 2006; 281:30561-30572.

38. Leren TP. Mutations in the PCSK 9 gene in Norwegian subjects with autosomal dominant hypercholesterolemia. Clin Genet. 2004; 65:419-422.

39. Timms KM, Wagner S, Samuels ME, Forbey K, Goldfine H, Jammulapati S, Skolnick MH, Hopkins PN, Hunt SC, Shattuck DM. A mutation in PCSK9 causing autosomaldominant hypercholesterolemia in a Utah pedigree. Hum Genet. 2004; 114:349-353.

40. Berge KE, Ose L, Leren TP. Missense mutations in the PCSK9 gene are associated with hypocholesterolemia and possibly increased response to statin therapy. Arterioscler Thromb Vasc Biol. 2006; 26:1094-1100.

41. Manthey GM, McEwen JE. The product of the nuclear gene PET309 is required for translation of mature mRNA and stability or production of intron-containing RNAs derived from the mitochondrial COX1 locus of Saccharomyces cerevisiae. EMBO J. 1995; 14:4031-4043.

42. Le Hir H, Nott A, Moore MJ. How introns influence and enhance eukaryotic gene expression. Trends Biochem Sci. 2003; 28:215-220.

43. Almeida OP, Alfonso H, Flicker L, Hankey GJ, Norman PE. Cardiovascular disease, depression and mortality: The Health In Men Study. Am J Geriatr Psychiatry. 2012; 20:433-440.

44. Chuang CS, Yang TY, Muo CH, Su HL, Sung FC, Kao $\mathrm{CH}$. Hyperlipidemia, statin use and the risk of developing depression: A nationwide retrospective cohort study. Gen Hosp Psychiatry. 2014; 36:497-501.

(Received September 19, 2017; Revised October 15, 2017; Accepted October 21, 2017) 